\title{
In-medium meson properties and field transformations
}

\author{
S. Kondratyuk, K. Kubodera, and F. Myhrer \\ Nuclear Theory Group, Department of Physics and Astronomy, \\ University of South Carolina, 712 Columbia, SC 29208, USA
}

(Dated: October 31, 2018)

\begin{abstract}
Since the existing calculations of the effective meson mass in nuclear medium involve approximations, it is important to examine whether they satisfy the general requirement of the equivalence theorem that the physical observables should be independent of the choice of field variables. We study here consequences of nucleon field transformations. As an illustrative case we consider the in-medium effective pion mass calculated for the s-wave pion-nucleon interaction in the linear density approximation. We demonstrate that it is necessary to include the Born term explicitly in order that the effective pion mass should obey the equivalence theorem.
\end{abstract}

PACS numbers: 14.40.Aq, 21.65.+f, 03.75.Kk, 12.39.Fe 


\section{INTRODUCTION}

Although the behavior of mesons in nuclear medium, including the possibility of meson condensation, has been extensively studied for several decades 11, 2, 3, 4], various aspects of the problem continue to draw considerable attention [5, 6, 7, 8, 9, 10]. A number of calculations of effective in-medium meson properties have been done based on effective field theory approaches, such as chiral perturbation theory $(\chi \mathrm{PT})$, in order to incorporate the basic properties of low-energy hadron interactions in a field-theoretic framework. One of the issues discussed intensively in the literature is the off-shell invariance of the in-medium effective meson mass (or, equivalently, its invariance under transformations of interpolating field variables). According to the well-known equivalence theorem 11, 12, 13, 14, 15] any observables, and hence in-medium observables as well, should be independent of the choice of field variables. A question of practical importance is whether this requirement is fulfilled in actual calculations that involve approximations such as truncations of the lagrangian, the linear-density approximation, etc. (see, e.g., 6, 7, 8, 9]). Detailed studies by Thorsson and Wirzba [7] and Lee et al. 8] have demonstrated that the in-medium effective meson mass calculated in the linear baryon density approximation and next-to-leading order in $\chi \mathrm{PT}$ is independent of the choice of meson field variables in conformity with the equivalence theorem. Beyond the linear-density approximation, one needs to take into account the possible influence of multi-baryonic terms in the lagrangian as well as nuclear correlations. This challenging problem has been addressed by several authors (see, e.g., Refs. [2, 8, 9, 10]), and further studies in this direction are certainly warranted.

To shed more light on the issue of the off-shell invariance of the in-medium meson properties, in this article we examine consequences of transformations of the nucleon field. Our study is supplementary to those of Refs. 6, 7, 8, 9] wherein transformations of meson fields were discussed. We limit ourselves here to the consideration of an in-medium effective pion mass calculated in the standard linear density approximation of the optical potential theory [1, 2, 16]. Furthermore, we only deal with the s-wave pion-nucleon interaction. Although (possible) meson condensation is very likely to be influenced by not only s-wave but also p-wave interactions, we believe it is still informative to study the s-wave contributions alone. While s-wave interactions are more relevant to kaon condensation [4], we consider here the pion for simplicity. This should not affect the main conclusions of this report since they follow from the transformation of the nucleon - rather than meson - field. The basic nature of the issues involved is expected to reveal itself even in the present limited treatment.

The pion-nucleon scattering amplitude is calculated here based on the relativistic chiral lagrangian [17] at tree level. In this formalism the in-medium modification of the pion mass comprises contributions from contact pion-nucleon interactions and from the nucleon exchange (Born) term. The contact interaction includes effects of explicit chiral symmetry breaking through the nucleon sigma-term and has been extensively considered in the past [6, 7]. In this paper we emphasize that, in addition to the contact interaction, the Born term must be explicitly taken into account in order to ensure the off-shell invariance of the effective pion mass. This is shown by evaluating the effective in-medium mass in two different representations of the lagrangian which are connected by a transformation of the nucleon interpolating field. 


\section{THE EFFECTIVE LAGRANGIAN}

Our arguments are based on the second order relativistic chiral lagrangian [17, 18] containing the pion field $\varphi_{\alpha}$ (with isovector index $\alpha=1,2,3$ ) and the nucleon field $\psi$. The lagrangian reads

$$
\mathcal{L}=\mathcal{L}_{\pi \pi}^{(2)}+\mathcal{L}_{\pi N}^{(1)}+\mathcal{L}_{\pi N}^{(2)}
$$

where the superscripts show the order in $Q$, with $Q$ being a generic notation for small fourmomenta or the pion mass. The pion lagrangian $\mathcal{L}_{\pi \pi}^{(2)}$ is given in terms of the standard non-linear fields, $U$, which we expand in powers of the pion field:

$$
\begin{gathered}
U=u^{2}=\exp \left(i \frac{\vec{\tau} \cdot \vec{\varphi}}{f}\right)=1+\frac{i}{f} \vec{\tau} \cdot \vec{\varphi}-\frac{1}{2 f^{2}} \vec{\varphi}^{2}+\cdots \\
u_{\mu}=i u^{\dagger}\left(\partial_{\mu} U\right) u^{\dagger}=-\frac{1}{f} \vec{\tau} \cdot \partial_{\mu} \vec{\varphi}+\cdots
\end{gathered}
$$

and

$$
\chi_{+}=M^{2}\left(U^{\dagger}+U\right)=2 M^{2}-\frac{M^{2}}{f^{2}} \vec{\varphi}^{2}+\cdots,
$$

where $f$ is the pion decay constant, $M$ is the pion mass and $\tau^{\alpha}$ are the Pauli isospin matrices. ${ }^{1}$ Since we will consider only tree-level contributions to the pion-nucleon scattering amplitude, we will omit polynomials containing three or more pion fields; such terms are denoted by an ellipsis. The purely pionic lagrangian in Eq. (11) is

$$
\mathcal{L}_{\pi \pi}^{(2)}=\frac{f^{2}}{4} \operatorname{Tr}\left(u_{\mu} u^{\mu}+\chi_{+}\right)=\frac{1}{2}\left(\partial_{\mu} \vec{\varphi}\right) \cdot\left(\partial^{\mu} \vec{\varphi}\right)-\frac{M^{2}}{2} \vec{\varphi}^{2}+\cdots
$$

with the trace pertaining to the $2 \times 2$ isospin matrices. The pion-nucleon lagrangian in Eq. (11) involves the covariant derivative

$$
D_{\mu} \psi=\left(\partial_{\mu}+\frac{1}{2}\left[u^{\dagger},\left(\partial_{\mu} u\right)\right]\right) \psi=\left(\partial_{\mu}+\frac{i}{4 f^{2}} \epsilon_{\alpha \beta \gamma} \tau^{\gamma} \varphi^{\alpha}\left(\partial_{\mu} \varphi^{\beta}\right)\right) \psi+\cdots,
$$

where we have used Eq. (2) for the expansion of $u$. The relevant parts of the lagrangian are

$$
\begin{aligned}
\mathcal{L}_{\pi N}^{(1)} & =\bar{\psi}(i \not D-m) \psi+\frac{1}{2} g_{A} \bar{\psi} \psi \gamma_{5} \psi \\
& =\bar{\psi}(i \not \partial-m) \psi-\frac{1}{4 f^{2}} \epsilon_{\alpha \beta \gamma} \bar{\psi} \tau^{\gamma} \varphi^{\alpha}\left(\not \partial \varphi^{\beta}\right) \psi-\frac{g_{A}}{2 f} \bar{\psi} \vec{\tau} \cdot(\not \partial \vec{\varphi}) \gamma_{5} \psi+\cdots
\end{aligned}
$$

and

$$
\begin{aligned}
\mathcal{L}_{\pi N}^{(2)}= & c_{1} \operatorname{Tr}\left(\chi_{+}\right) \bar{\psi} \psi-\frac{c_{2}}{4 m^{2}} \operatorname{Tr}\left(u_{\mu} u_{\nu}\right)\left(\bar{\psi} D^{\mu} D^{\nu} \psi+\text { herm. conj. }\right) \\
& +\frac{c_{3}}{2} \operatorname{Tr}\left(u_{\mu} u^{\mu}\right) \bar{\psi} \psi-\frac{c_{4}}{4} \bar{\psi} \gamma^{\mu} \gamma^{\nu}\left[u_{\mu}, u_{\nu}\right] \psi \\
= & c_{1}\left(4 M^{2}-\frac{2 M^{2}}{f^{2}} \vec{\varphi}^{2}\right) \bar{\psi} \psi-\frac{c_{2}}{2 m^{2} f^{2}}\left(\partial_{\mu} \vec{\varphi}\right) \cdot\left(\partial_{\nu} \vec{\varphi}\right) \bar{\psi}\left(\partial^{\mu} \partial^{\nu} \psi\right) \\
& -\frac{c_{2}}{2 m^{2} f^{2}}\left(\partial_{\mu} \bar{\varphi}\right) \cdot\left(\partial_{\nu} \vec{\varphi}\right)\left(\partial^{\mu} \partial^{\nu} \bar{\psi}\right) \psi+\frac{c_{3}}{f^{2}}\left(\partial_{\mu} \vec{\varphi}\right) \cdot\left(\partial^{\mu} \vec{\varphi}\right) \bar{\psi} \psi \\
& -i \epsilon_{\alpha \beta \gamma} \frac{c_{4}}{2 f^{2}} \bar{\psi} \tau^{\gamma}\left(\not \partial \varphi^{\alpha}\right)\left(\not \partial \varphi^{\beta}\right) \psi+\cdots
\end{aligned}
$$

\footnotetext{
${ }^{1}$ We will put arrows over isospin vectors and use the boldface for the usual three-vectors.
} 
where $m$ is the nucleon mass, $g_{A}$ is the axial coupling constant and $c_{1}, \cdots, c_{4}$ are the standard low energy constants. ${ }^{2}$

It is convenient to combine $-m \bar{\psi} \psi$ from Eq. (17) with $4 c_{1} M^{2} \bar{\psi} \psi$ from Eq. (8) to form a modified nucleon mass $m_{N}=m-4 c_{1} M^{2}$. The difference between $m_{N}$ and $m$ will lead to corrections of $\mathcal{O}\left(Q^{4}\right)$ in all the quantities calculated below. Since such corrections are beyond the order $\mathcal{O}\left(Q^{2}\right)$ we are interested in, we will simply keep the notation $m$ for the nucleon mass and drop the term $4 c_{1} M^{2} \bar{\psi} \psi$ from $\mathcal{L}_{\pi N}^{(2)}$.

\section{EFFECTIVE PION MASS IN NUCLEAR MATTER}

The effective in-medium pion mass is determined by the pole position of the full inmedium pion propagator 3]. The pole position corresponds to the energy of a pionic mode state and is related to the pion self-energy in the medium. We will apply the usual mean-field approximation 2, 16], where, to first order in the nuclear density $\rho$, the s-wave pion selfenergy is proportional to the isospin-symmetric forward pion-nucleon scattering amplitude. The general structure of the pion-nucleon scattering amplitude is [21]

$$
T_{\alpha \beta}\left(p, q ; p^{\prime}, q^{\prime}\right)=\bar{u}\left(p^{\prime}\right)\left[\delta_{\alpha \beta}\left(D^{+}+\frac{\left[\not, \phi^{\prime}\right]}{4 m} B^{+}\right)+\frac{\left[\tau_{\alpha}, \tau_{\beta}\right]}{2}\left(D^{-}+\frac{\left[\phi, \phi^{\prime}\right]}{4 m} B^{-}\right)\right] u(p),
$$

where the initial and final nucleon and pion four-momenta are $p, p^{\prime}$ and $q, q^{\prime}$, respectively, and the initial and final pion isospin indices are $\alpha$ and $\beta$. The nucleons are on the mass shell - described by Dirac spinors $u(p), \bar{u}\left(p^{\prime}\right)$ - while the pions can be off-shell in general. The amplitudes $D^{ \pm}$and $B^{ \pm}$are functions of the standard kinematic variables

$$
\nu=\frac{q^{2}-q^{2}+2\left(p\left(q+q^{\prime}\right)\right)}{4 m}, \quad \nu_{B}=-\frac{\left(q q^{\prime}\right)}{2 m} .
$$

The isospin-symmetric, s-wave interaction is described by the invariant amplitude $D^{+}$. In the tree-level approximation, the amplitude comprises contributions from the s- and u-channel nucleon pole graphs, which constitute the "pseudovector" Born term $D_{\text {Born }}^{+}=$ $D_{s}^{+}+D_{u}^{+}$, and from the four-particle contact graph, $D_{c}^{+}$:

$$
D^{+}=D_{\text {Born }}^{+}+D_{c}^{+}=D_{s}^{+}+D_{u}^{+}+D_{c}^{+}
$$

where the explicit expressions are obtained using the lagrangians Eqs. (17) 8):

$$
\begin{aligned}
D_{s}^{+} & =\frac{m g_{A}^{2}}{2 f^{2}}\left(\frac{\nu_{B}}{\nu_{B}-\nu}-\frac{\nu}{2 m}\right) \\
D_{u}^{+} & =\frac{m g_{A}^{2}}{2 f^{2}}\left(\frac{\nu_{B}}{\nu_{B}+\nu}+\frac{\nu}{2 m}\right), \\
D_{c}^{+} & =-\frac{4 c_{1} M^{2}}{f^{2}}+\frac{c_{2}}{m^{2} f^{2}}\left[(q p)\left(q^{\prime} p\right)+\left(q p^{\prime}\right)\left(q^{\prime} p^{\prime}\right)\right]+\frac{2 c_{3}}{f^{2}}\left(q q^{\prime}\right)
\end{aligned}
$$

\footnotetext{
${ }^{2}$ We use the definitions of [7, 19] for the low-energy constants, and the conventions of [20] for the Dirac matrices, Lorentz vectors and their products, Feynman rules, etc.
} 
In the mean-field approximation [2, 16] the pion self-energy $\Pi(\omega, \mathbf{k}, \rho)$ is given by the forward scattering amplitude $D^{+}$in the nucleon rest frame, i.e., for $\nu$ and $\nu_{B}$ evaluated from Eqs. (10) with $p=p^{\prime}=(m, \mathbf{0})$ and $q=q^{\prime}=(\omega, \mathbf{k})$ :

$$
\Pi(\omega, \mathbf{k}, \rho)=-\rho D^{+}\left(\nu=\omega, \nu_{B}=\left(\mathbf{k}^{2}-\omega^{2}\right) /(2 m)\right) .
$$

This kinematical situation describes a pion interacting with a heavy, non-recoiling nucleon in the Fermi sea. Substitution of Eqs. (11) 14) into Eq. (15) yields

$$
\Pi(\omega, \mathbf{k}, \rho)=\frac{\rho}{f^{2}}\left[\frac{g_{A}^{2}\left(\mathbf{k}^{2}-\omega^{2}\right)^{2}}{4 m \omega^{2}}-\sigma-2\left(c_{2}+c_{3}\right) \omega^{2}+2 c_{3} \mathbf{k}^{2}\right]+\mathcal{O}\left(Q^{3}, \rho^{2}\right),
$$

where $\sigma=-4 c_{1} M^{2}$ equals the nucleon sigma-term up to corrections of $\mathcal{O}\left(Q^{3}\right)$ [19].

The effective in-medium pion mass $M_{e f f}$ is defined as the pole of the pion propagator

$$
\left(\omega^{2}-\mathbf{k}^{2}-M^{2}-\Pi(\omega, \mathbf{k}, \rho)\right)^{-1}
$$

when $\mathbf{k}=\mathbf{0}$, i.e., $M_{e f f}$ is the solution of the dispersion equation for $\omega$ :

$$
\omega^{2}-M^{2}-\Pi(\omega, \mathbf{k}=\mathbf{0}, \rho)=0 .
$$

Using the pion self-energy of Eq. (16), we get for the square of the effective mass

$$
\begin{aligned}
M_{e f f}^{2} & =M^{2}+M_{e f f, B o r n}^{2}+M_{e f f, c}^{2}+\mathcal{O}\left(Q^{3}, \rho^{2}\right) \\
& =M^{2}\left[1-\frac{\rho}{f^{2} M^{2}}\left(-\frac{g_{A}^{2} M^{2}}{4 m}+\sigma+2\left(c_{2}+c_{3}\right) M^{2}\right)\right]+\mathcal{O}\left(Q^{3}, \rho^{2}\right),
\end{aligned}
$$

where we have separated the Born contribution for later convenience. The contribution of the Born term, $D_{\text {Born }}^{+}$, to $M_{e f f}^{2}$ is

$$
M_{e f f, B o r n}^{2}=\rho \frac{g_{A}^{2} M^{2}}{4 m f^{2}}
$$

while the contribution of the contact term $D_{c}^{+}$equals

$$
M_{e f f, c}^{2}=-\frac{\rho}{f^{2}}\left[\sigma+2\left(c_{2}+c_{3}\right) M^{2}\right] .
$$

The Born contribution is estimated to be $M_{\text {eff,Born }}^{2} \approx 0.066 M^{2}\left(\rho / \rho_{0}\right)$, where $\rho_{0}$ is the normal nuclear matter density $\left(\rho_{0}=0.17 \mathrm{fm}^{-3}\right)$, using $g_{A}=1.27, f=92.4 \mathrm{MeV}, M=139 \mathrm{MeV}$, $m=938 \mathrm{MeV}$. However, a quantitative evaluation of Eq. (19) is quite sensitive to the precise values of the sigma-term $\sigma$ and the low-energy constants $c_{2}$ and $c_{3}$ (see Refs. [19, 22] regarding the values of the low-energy constants, and Refs. 23] for a recent discussion on the sigma-term). Such numerical analysis is outside the scope of this work, and we remark only that the Born and the contact term contributions to $M_{\text {eff }}^{2}$ are of the same order, $\mathcal{O}\left(Q^{2}\right)$.

At first sight, the presence of the Born contribution $M_{e f f, B o r n}^{2}$ in Eq. (19) may not look significant, as its effect can formally be absorbed into the contact term by using, e.g., $c_{2}^{\prime}=c_{2}-g_{A}^{2} /(8 m)$ instead of $c_{2}$. In fact, the expression $M_{\text {eff }}^{2}=M^{2}+M_{e f f, c}^{2}$ has appeared in the literature [7]. We shall show however that, in order to ensure that $M_{\text {eff }}^{2}$ is independent of the nucleon interpolating field, one must keep track of the terms of different origins. 


\section{TRANSFORMATION OF THE NUCLEON FIELD AND THE EQUIVA- LENCE THEOREM FOR THE IN-MEDIUM PION MASS}

Insofar as the effective pion mass has the status of an observable quantity, the equivalence theorem requires it to be invariant with respect to redefinitions of the interpolating - or offshell - fields. The off-shell invariance of the scattering matrix has been studied in various approaches, including axiomatic field theory [11], lagrangian and hamiltonian formalisms [12, 13], the path-integral method [14] (which in $\chi \mathrm{PT}$ is exemplified by the generating functional approach [17, 24]) and Feynman-graph techniques [15]. The role of the equivalence theorem in hadronic physics has been also discussed recently within several models and approximation schemes [25].

The main purpose of the present paper is to study consequences of the equivalence theorem for the effective in-medium pion mass as calculated using the commonly employed mean-field approximation. It should be emphasized that the off-shell invariance of observables holds not only in general, but also at tree level separately [13]. This fact is crucial for our present calculation as it allows us to apply the equivalence theorem to the tree-level amplitudes, which significantly simplifies the approach. It is also important that the equivalence theorem is applicable to the transformations involving only the pion or only the nucleon fields, as well as to more complicated transformations in which both pion and nucleon field variables change. The invariance of the effective mass with respect to field transformations of the pion field separately was extensively discussed before (see, e.g., Refs. [6, 7, 8, [9]). Here we consider a complementary case where it is the nucleon field that undergoes a transformation while the pion field does not change. Specifically, we consider a transformation of the nucleon field

$$
\psi \longrightarrow\left(1+i \frac{\lambda}{f} \gamma_{5} \vec{\tau} \cdot \vec{\varphi}-\frac{\lambda^{2}}{2 f^{2}} \vec{\varphi}^{2}\right) \psi
$$

where $\lambda$ is a real parameter. This may be viewed as a truncated form of the chiral rotation $\psi \rightarrow \exp \left\{i(\lambda / f) \gamma_{5} \vec{\tau} \cdot \vec{\varphi}\right\} \psi$ in which the terms linear and quadratic in the pion field are retained. As before, we drop the terms with three or more pion fields since they are irrelevant to our tree-level calculation. The truncation, however, should not suggest that the parameter of the transformation is small: the equivalence theorem holds for any $\lambda$ in Eq. (22). The redefinition of the nucleon field Eq. (22) entails the transformation of the lagrangian in Eq. (77):

$$
\begin{aligned}
\mathcal{L}_{\pi N}^{(1)} \longrightarrow & \mathcal{L}_{\pi N}^{(1)}-\frac{\lambda}{f} \bar{\psi} \vec{\tau} \cdot(\not \partial \vec{\varphi}) \gamma_{5} \psi-i \frac{2 m \lambda}{f} \bar{\psi} \vec{\tau} \cdot \vec{\varphi} \gamma_{5} \psi+\frac{g_{A} \lambda}{f^{2}} \epsilon_{\alpha \beta \gamma} \bar{\psi}\left(\not \partial \varphi^{\alpha}\right) \varphi^{\beta} \tau^{\gamma} \psi \\
& -\epsilon_{\alpha \beta \gamma} \frac{\lambda^{2}}{f^{2}} \bar{\psi} \varphi^{\alpha}\left(\not \partial \varphi^{\beta}\right) \tau^{\gamma} \psi+\frac{2 m \lambda^{2}}{f^{2}} \vec{\varphi}^{2} \bar{\psi} \psi+\cdots
\end{aligned}
$$

while the lagrangians $\mathcal{L}_{\pi \pi}^{(2)}$ and $\mathcal{L}_{\pi N}^{(2)}$ in Eqs. (5, 8) do not change. The transformed lagrangian, given by the sum of Eqs. (15, 23, 8), defines a new representation of the theory; we will put tildes over the quantities calculated from the transformed lagrangian.

The tree-level isospin-symmetric amplitude in the new representation is the sum of a new Born term, $\widetilde{D}_{\text {Born }}^{+}=\widetilde{D}_{s}^{+}+\widetilde{D}_{u}^{+}$, and a new contact term, $\widetilde{D}_{c}^{+}$:

$$
\widetilde{D}^{+}=\widetilde{D}_{\text {Born }}^{+}+\widetilde{D}_{c}^{+}=\widetilde{D}_{s}^{+}+\widetilde{D}_{u}^{+}+\widetilde{D}_{c}^{+}
$$


Using the transformed lagrangian, we obtain for the new s-channel, u-channel and contact diagrams:

$$
\begin{aligned}
& \widetilde{D}_{s}^{+}=\frac{m g_{A}^{2}}{2 f^{2}}\left(\frac{\nu_{B}}{\nu_{B}-\nu}-\frac{\nu}{2 m}\right)-\frac{\lambda^{2}(2 m+\nu)+\lambda g_{A} \nu}{f^{2}} \\
& \widetilde{D}_{u}^{+}=\frac{m g_{A}^{2}}{2 f^{2}}\left(\frac{\nu_{B}}{\nu_{B}+\nu}+\frac{\nu}{2 m}\right)-\frac{\lambda^{2}(2 m-\nu)-\lambda g_{A} \nu}{f^{2}} \\
& \widetilde{D}_{c}^{+}=-\frac{4 c_{1} M^{2}}{f^{2}}+\frac{c_{2}}{m^{2} f^{2}}\left[(q p)\left(q^{\prime} p\right)+\left(q p^{\prime}\right)\left(q^{\prime} p^{\prime}\right)\right]+\frac{2 c_{3}}{f^{2}}\left(q q^{\prime}\right)+\frac{4 m \lambda^{2}}{f^{2}} .
\end{aligned}
$$

Note that both $\widetilde{D}_{\text {Born }}^{+}=\widetilde{D}_{s}^{+}+\widetilde{D}_{u}^{+}$and $\widetilde{D}_{c}^{+}$depend on the arbitrary transformation parameter $\lambda$. Only the sum $\widetilde{D}^{+}=\widetilde{D}_{B \text { orn }}^{+}+\widetilde{D}_{c}^{+}$is independent of $\lambda$. Thus the complete tree-level scattering amplitude is invariant under the field transformation Eq. (22) and we have:

$$
\widetilde{D^{+}}=D^{+}
$$

Exactly as was done in the original representation (see Eqs. (15, 18), the effective mass $\widetilde{M}_{e f f}$ in the new representation is obtained by solving the dispersion equation

$$
\omega^{2}-M^{2}-\widetilde{\Pi}(\omega, \mathbf{k}=\mathbf{0}, \rho)=0,
$$

where the pion self-energy is proportional to the amplitude $\widetilde{D}^{+}$evaluated at the forward scattering, nucleon rest frame kinematics:

$$
\widetilde{\Pi}(\omega, \mathbf{k}, \rho)=-\rho \widetilde{D}^{+}\left(\nu=\omega, \nu_{B}=\left(\mathbf{k}^{2}-\omega^{2}\right) /(2 m)\right) .
$$

Using Eqs. (24 27), we obtain in the new representation:

$$
\widetilde{M}_{e f f}^{2}=M^{2}+\widetilde{M}_{e f f, B o r n}^{2}+\widetilde{M}_{e f f, c}^{2}+\mathcal{O}\left(Q^{3}, \rho^{2}\right),
$$

where the Born and the contact term contributions equal

$$
\widetilde{M}_{e f f, B o r n}^{2}=\frac{\rho}{f^{2}}\left(\frac{g_{A}^{2} M^{2}}{4 m}+4 m \lambda^{2}\right)
$$

and

$$
\widetilde{M}_{e f f, c}^{2}=-\frac{\rho}{f^{2}}\left[\sigma+2\left(c_{2}+c_{3}\right) M^{2}+4 m \lambda^{2}\right],
$$

respectively. The $\lambda$-dependent terms present in $\widetilde{M}_{\text {eff,Born }}^{2}$ and $\widetilde{M}_{\text {eff,c }}^{2}$ mutually cancel when the sum in Eq. (31) is taken, and comparison with Eq. (19) shows that the equivalence theorem is fulfilled for the effective mass:

$$
\widetilde{M}_{e f f}=M_{e f f}
$$

which is a direct consequence of Eq. (28). The crucial observation is that this representation invariance of the in-medium pion mass holds only provided both the Born and the contact contributions are taken into account.

Without considering the nucleon field transformation, one might falsely conclude from Eq. (19) that the effective mass could be calculated based on the contact term alone. For 
example, from the outset one might want to use $c_{2}^{\prime}=c_{2}-g_{A}^{2} /(8 m)$ instead of $c_{2}$ in the lagrangian $\mathcal{L}_{\pi N}^{(2)}$ of Eq. (8) in order to forgo the Born term throughout the calculation. In doing so, one would have to take into account the following two points. First, the consistency of calculation would require one to drop the Born term in all considered representations. Second, since the equivalence theorem dictates [12, 15] that transitions between representations are effected solely by changes of field variables while the coupling constants must stay unchanged, the constant $c_{2}$ could not be redefined differently in different representations. In the original representation, a calculation based on the contact term with the constant $c_{2}^{\prime}$ would lead to an effective mass of the form

$$
M^{2}\left[1-\frac{\rho}{f^{2} M^{2}}\left(\sigma+2\left(c_{2}^{\prime}+c_{3}\right) M^{2}\right)\right]+\mathcal{O}\left(Q^{3}, \rho^{2}\right),
$$

which numerically gives the same value as Eq. (19). However, an effective mass calculated solely from the corresponding contact term in the new representation would then equal

$$
M^{2}\left[1-\frac{\rho}{f^{2} M^{2}}\left(\sigma+2\left(c_{2}^{\prime}+c_{3}\right) M^{2}\right)\right]-\rho \frac{4 m \lambda^{2}}{f^{2}}+\mathcal{O}\left(Q^{3}, \rho^{2}\right) .
$$

This expression contains an arbitrary $\lambda$-dependent term $-\rho 4 m \lambda^{2} / f^{2}$ and hence cannot in general be equal to Eq. (35). Thus any attempt to mimic the Born contribution by modifying the contact term will lead to an effective mass which will depend on an unphysical parameter, which is unacceptable since $M_{\text {eff }}$ is an observable quantity that should not depend on the choice of representation.

\section{CONCLUDING REMARKS}

In an approach based on the relativistic chiral lagrangian, we have shown that it is impossible to have a representation-invariant in-medium pion mass by considering the pionnucleon contact interactions alone. We have also demonstrated that the representation invariance cannot be obtained by subsuming the nucleon-exchange effects (as represented by the Born term) into the contact term. Only by explicitly including the Born contribution, together with the contact term, does the effective mass become invariant under redefinitions of the interpolating nucleon field.

As a physical observable, the effective mass should be independent of the choice of all interpolating fields of the lagrangian. The relativistic chiral lagrangian employed in the present work at tree level provides a suitable model for studying effects of nucleon field transformations. It would be interesting to obtain analogous results within the framework of non-relativistic $\chi \mathrm{PT}$ which up to now has been applied only in connection with meson field transformations [6, 7, 8, 9]. In contrast to the relativistic approach with the pion-nucleon lagrangians Eqs. (7) 8) in the original representation, or with their counterparts Eqs. (23, 8) in the new representation, the non-relativistic heavy-baryon formalism using the standard dimensional regularization would allow for a one-to-one correspondence between the loop and small momentum expansions [17, 19, 26]. Another line of development would be to consider consequences of simultaneous changes of meson and nucleon field variables.

To make our argument as transparent as possible, we adopted in the present paper the mean-field approximation limited to leading order in the nuclear density and considered s-wave interactions only. Nevertheless, our main conclusion - that in calculating in-medium 
meson properties it is necessary to include the meson-nucleon Born contribution explicitly in addition to the contact interaction - follows from quite general field-theoretical considerations and should therefore hold in more realistic models as well.

\section{Acknowledgments}

We thank Youngman Kim for useful discussions. This work is supported in part by the US National Science Foundation, Grant Nos. PHY-9900756 and PHY-0140214.

[1] L. S. Kisslinger, Phys. Rev. 98, 761 (1955).

[2] M. Ericson and T. E. O. Ericson, Ann. Phys. (NY) 36, 323 (1966).

[3] A. B. Migdal, Zh. Eksp. Teor. Fiz. 61, 2209 (1971) [Sov. Phys. JETP 34, 1184 (1972)]; Phys. Rev. Lett. 31, 257 (1973); R. F. Sawyer, Phys. Rev. Lett. 29, 382 (1972); D. J. Scalapino, Phys. Rev. Lett. 29, 386 (1972); B. H. Wilde, S. A. Coon, and M. D. Scadron, Phys. Rev. D 18, 4489 (1978).

[4] D. B. Kaplan and A. E. Nelson, Phys. Lett. B 175, 57 (1986); G. E. Brown, K. Kubodera, and M. Rho, Phys. Lett. B 192, 273 (1987).

[5] H. Yabu, S. Nakamura, and K. Kubodera, Phys. Lett. B 317, 269 (1993); H. Yabu, S. Nakamura, F. Myhrer, and K. Kubodera, Phys. Lett. B 315, 17 (1993).

[6] H. Yabu, F. Myhrer, and K. Kubodera, Phys. Rev. D 50, 3549 (1994).

[7] V. Thorsson and A. Wirzba, Nucl. Phys. A 589, 633 (1995).

[8] C.-H. Lee, G. E. Brown, D.-P. Min, and M. Rho, Nucl. Phys. A 585, 401 (1995).

[9] T.-S. Park, H. Jung, D.-P. Min, J. Kor. Phys. Soc. 41, 195 (2002).

[10] V. R. Pandharipande, C. J. Pethick, and V. Thorsson, Phys. Rev. Lett. 75, 4567 (1995); N. Kaiser and W. Weise, Phys. Lett. B 512, 283 (2001); Ulf.-G. Meissner, J. A. Oller, and A. Wirzba, Ann. Phys. (NY) 297, 27 (2002); T. Muto, Nucl. Phys. A 697, 225 (2002); E. E. Kolomeitsev and D. Voskresensky, nucl-th/0211052; T. Tatsumi, nucl-th/0302009.

[11] R. Haag, Phys. Rev. 112, 669 (1958); O. W. Greenberg, Phys. Rev. 115, 706 (1959); H. Ekstein, Phys. Rev. 117, 1590 (1960); H.-J. Borchers, Nuovo Cimento 15, 784 (1960); R. F. Streater and A. S. Wightman, PCT, Spin and Statistics, and All That (W. A. Benjamin, Inc., London 1964); N. N. Bogolubov, A. A. Logunov, and I. T. Todorov, Introduction to Axiomatic Quantum Field Theory (W. A. Benjamin, Inc., London 1975); R. Haag, Local Quantum Physics: Fields, Particles, Algebras (Springer, Berlin 1992).

[12] J. S. R. Chisholm, Nucl. Phys. 26, 469 (1961); S. Kamefuchi, L. O'Raifeartaigh, and A. Salam, Nucl. Phys. 28, 529 (1961);

[13] S. Coleman, J. Wess, and B. Zumino, Phys. Rev. 177, 2239 (1969).

[14] A. Salam and J. Strathdee, Phys. Rev. D 2, 2869 (1970).

[15] Y.-M. P. Lam, Phys. Rev. D 7, 2943 (1973); G. 't Hooft and M. Veltman, Diagrammar (CERN Yellow Report 73-09).

[16] B. D. Serot and J. D. Walecka, Adv. Nucl. Phys. 16, 1 (1986); J. Delorme, M. Ericson, and T. E. O. Ericson, Phys. Lett. B 291, 379 (1992).

[17] J. Gasser, M. E. Sainio, and A. Svarc, Nucl. Phys. B 307, 779 (1988). 
[18] T. Becher and H. Leutwyler, Eur. Phys. J. C 9, 643 (1999); T. Becher and H. Leutwyler, JHEP 0106, 017 (2001).

[19] V. Bernard, N. Kaiser, and Ulf.-G. Meissner, Phys. Lett. B 309, 421 (1993); V. Bernard, N. Kaiser, and Ulf.-G. Meissner, Int. J. Mod. Phys. E 4, 193 (1995).

[20] J. D. Bjorken and S. D. Drell, Relativistic Quantum Mechanics (McGraw-Hill, 1964).

[21] G. Höhler, Pion-Nucleon Scattering, Landolt-Börnstein, 1/9b2, ed. H. Schopper (Springer, 1983).

[22] N. Fettes and Ulf-G. Meissner, Nucl. Phys. A 676, 311 (2000).

[23] M. E. Sainio, $\pi N$ Newslett. 16, 138 (2002); M. M. Pavan, I. I. Strakovsky, R. L. Workman, and R. A. Arndt, $\pi N$ Newslett. 16, 110 (2002).

[24] J. Gasser and H. Leutwyler, Ann. Phys. (NY) 158, 142 (1984); H. Leutwyler, Ann. Phys. (NY) 235, 165 (1994).

[25] S. Scherer and H. W. Fearing, Phys. Rev. C 51, 359 (1995); R. M. Davidson and G. I. Poulis, Phys. Rev. D 54, 2228 (1996); J. Adam, F. Gross, and J. W. Van Orden, Few Body Syst. 25, 73 (1998); H. W. Fearing and S. Scherer, Phys. Rev. C 62, 034003 (2000); R. J. Furnstahl, H.-W. Hammer, and N. Tirfessa, Nucl. Phys. A 689, 846 (2001); S. Kondratyuk, A. D. Lahiff, H. W. Fearing, Phys. Lett. B 521, 204 (2001); B. Krippa, M. C. Birse, J. A. McGovern, and N. R. Walet, Phys. Rev. C 67, 031301 (2002).

[26] E. Jenkins and A. V. Manohar, Phys. Lett. B 255, 558 (1991). 